\title{
Calculating the Effective Capacitance for the RC Interconnect in VDSM Technologies
}

\author{
Soroush Abbaspour and Massoud Pedram \\ Department of Electrical Engineering-Systems \\ University of Southern California, Los Angeles, CA 90089 \\ \{sabbaspo, pedram\}@usc.edu
}

\begin{abstract}
In this paper, we present a new technique for calculating an effective capacitance of an RC interconnect line in very deep submicron design technologies. The calculation scheme guarantees that the effective capacitance model simultaneously matches both the $50 \%$ propagation delay and the $0-$ to- $0.8 V_{d d}$ output transition behavior of a standard cell driving an $R C$ interconnect. Experimental results show that the new technique exhibits high accuracy (less than 5\% error) and high efficiency (converges in two or at most three iterations). The paper also includes extensions to handle complex cells as drivers of the $R C$ interconnect.
\end{abstract}

\section{Introduction}

Circuit delay in VLSI circuits consists of two components: the $50 \%$ propagation delay of the driving gates (known as the gate propagation delays) and the delays of electrical signals through the wires (known as the interconnect propagation delays.) Consider the circuit in Fig.1. The overall delay from input pin "A" of gate "Inv1" to input pin "C" of "Inv 2" can be written as the sum of a gate propagation delay from input pin " $A$ " to the output pin "B" of "Inv1" and an interconnect propagation delay from output pin "B" to the input pin "C" of "Inv2."

$$
\text { Delay }_{A C}=\text { Delay }_{A B}+\text { Delay }_{B C}
$$

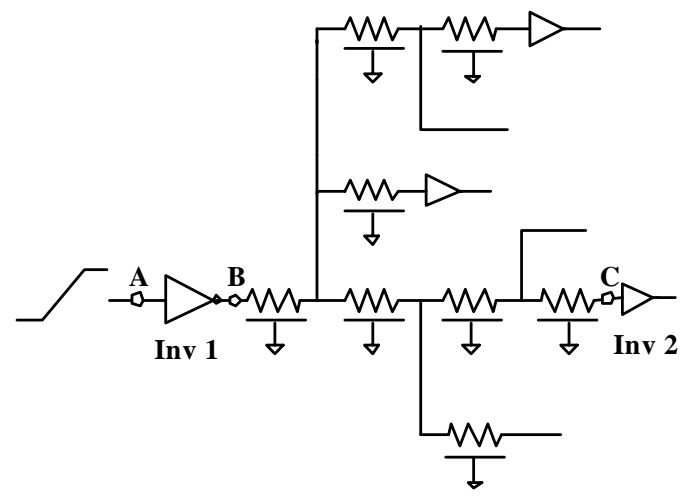

Figure 1: General delay model of interconnects which is driven by CMOS gates and driving another CMOS gates.

This work was supported by the National Science Foundation under grant number 9988441.
The gate propagation delay is in turn divided into two terms: the intrinsic gate delay and the (external) gate load delay. The intrinsic gate delay is due to the native characteristics of the CMOS devices (e.g., transistors) in the gates/cells (more precisely, it is equal to gate propagation delay under zero load condition.) The load delay captures the timing effect of the load on the gate propagation delay.

Fig. 2 shows a gate, which drives a purely capacitive load $\left(C_{\text {load }}\right)$ where one of its inputs switches (with a signal transition time of $T_{i n}$ ) and causes the output of the gate/cell to change. The gate propagation delay is a function of the input transition time and the output load. More precisely, the delay increases when $C_{\text {load }}$ and/or $T_{i n}$ increase, therefore,

$$
\text { Gate Delay }=f\left(T_{\text {in }}, C_{\text {load }}\right)
$$

Two approaches for gate propagation delay computation are based on (1) delay tables, and (2) use of a Thevenin equivalent circuit composed of a voltage source and a resistance in series with the gate load. Although the first approach is currently in wide use especially in the ASIC design flow, the second approach promises to be more accurate when the load is not purely capacitive. This is because it directly captures the interaction between the load and the gate/cell structure. The resistance value in the Thevenin model is strongly dependent on the input slew and output load and requires output voltage fitting [2].

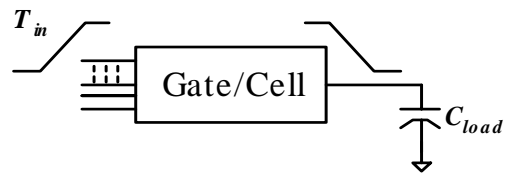

Figure 2: A gate/cell where its output switches when its input switches. The gate load delay is a function of both input slew and output load.

In commercial ASIC cell libraries, for each gate/cell in the library, typically, there is at least one pair of two-dimensional tables, which store the gate propagation delay and output rise/fall time as a function of the output capacitance and input slew. One sample delay table is shown in Fig. 3.

For intermediate values of input transition time and output load, piecewise linear approximation is used. The table is equivalent to empirical "k-factor' formulas for delay and output transition time. However, in VDSM technologies, we cannot neglect the effect of interconnect resistances and as shown in Fig. 1, the load is not purely capacitive. Using the sum of all load capacitances 
as the capacitive load is the simplest pessimistic approximation [7]. A better approximation for the n'th order load (i.e., with $n$ distributed capacitances) seen by the gate/cell is a second order RC- $\pi$ model $[3,5]$.

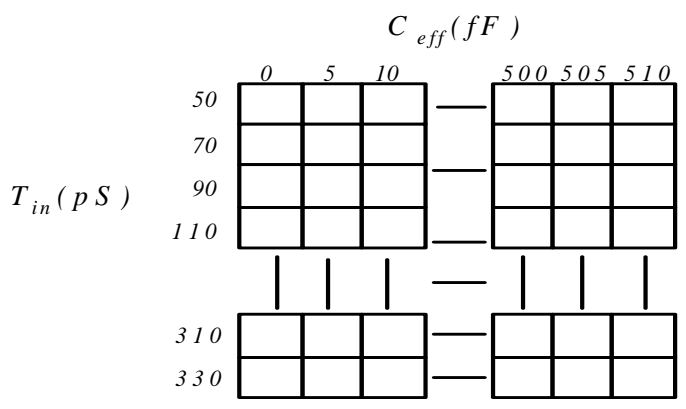

Figure 3: A two-dimensional delay table, one dimension is effective load and the other one is input transition time.

Equating the first, second and third moments of the admittance of the real load with the first, second and third moments of the RC- $\pi$ load, we can find $C_{1}, R_{\pi}$ and $C_{2}$ in Fig. 4.

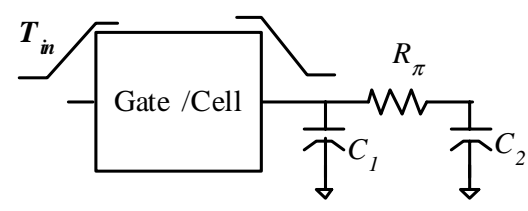

Figure 4: A gate/cell, which drives a RC- $\pi$ calculated load.

$Y_{\text {in }}(s)=A_{1} s+A_{2} s^{2}+A_{3} s^{3}+\ldots$.

$\hat{Y}_{\text {in }}(s)=\left(C_{1}+C_{2}\right) s-R_{\pi} C_{2}^{2} s^{2}+R_{\pi}^{2} C_{2}^{3} s^{3}+\ldots .$.

Eq. (3) is the Taylor expansion of the real load around $s=0$ and Eq. (4) is the Taylor expansion of the approximated load around $s=0$. Then,

$$
C_{1}=A_{1}-\frac{A_{2}^{2}}{A_{3}} \quad R_{\pi}=-\frac{A_{3}^{2}}{A_{2}{ }^{3}} \quad C_{2}=\frac{A_{2}^{2}}{A_{3}}
$$

It follows that for accurate delay calculation; we need to have a four-dimensional delay table, the four dimensions being input transition times, $C_{1}, R_{\pi}$, and $C_{2}$. However, this is very costly in terms of storage and computational requirements. Therefore, the "effective capacitance" approach was proposed in $[4,8]$. Effective capacitance approach uses the two-dimensional delay table; it approximates the $R C$ - $\pi$ model with an equivalent capacitance, which approximates the either $50 \%$ propagation delay or the transition time.

Given the problem described above, we propose an algorithm, which calculates an effective capacitance that results in a very good match both in terms of the output transition time and the $50 \%$ propagation delay, simultaneously. This paper is organized as follows: Section 2 reviews the previous work to compute effective capacitance. Section 3 describes our algorithm for cases of a simple driver. Section 4 extends the effective capacitance computation algorithm for complex gates. We provide the results in Section 5. The conclusions are presented in Section 6.

\section{Prior Work}

Many research results have been reported for calculating the interconnect propagation delay. These papers are simulationbased $[6,7,9]$ or rely on analytical derivations $[1,11]$. Similarly and more recently, a number of research results have been published that focus on the loading effect of the RC wires on the gate propagation delay $[8,9,12,13]$.

The effective capacitance is a function of two factors: (1) the output voltage waveform of the driving cell and (2) the load or more appropriately, the driving point admittance of interconnects. If two cells produce the same output waveform when the same load is applied, the two cells are equivalent in terms of calculating $C_{e f f}[12]$.

Consider the circuit in Fig. 4. If $R_{\pi}$ goes to infinity, then the gate/cell will see only $C_{1}$ as its load. On the other hand if $R_{\pi}$ goes to 0 , the gate/cell will see $C_{\text {tot }}=C_{1}+C_{2}$. Then the effective capacitance that the driver see, can be written as:

$$
C_{\text {eff }}=C_{1}+k C_{2} \quad \text { where } \quad 0 \leq k \leq 1
$$

Using a table of circuit simulation results and a pair of twodimensional delay tables (cf. Fig. 3), Macys et al. [12] obtain the effective capacitance. In their work, the effective capacitance is a function of the total capacitance in the RC- $\pi$ model $\left(C_{t o t}\right)$, the gate output slew rate, and the Elmore delay [1] of the load. The authors approximate the RC- $\pi$ load with an effective capacitance such that the output voltage waveforms of the driving cell passes through some critical voltages (e.g., 0 and $0.75 V_{d d}$ ) at the same instances in time. They also normalize the four model parameters (output slew time and three $\pi$ model parameters) to two parameters and use the table of circuit simulation results to find the effective capacitance by performing some iteration. However, their approach is not based on any analytical derivation. Furthermore, it requires a rather large number of iterations to achieve convergence. Finally, it is either inaccurate or very costly for complex gates.

In one extension to handle complex gate, Macys's approach can be modified where a complex gate is first converted into an equivalent inverter with an equivalent input slew time and then the delay tables are used to calculate the gate delay. This is obviously very inaccurate. In another extension, Macys's approach can be modified by generating a pair of twodimensional delay tables for each unique combination of possible input transitions and slew rates. Obviously, this is very expensive for complex gate with say three of four inputs. In contrast, in our approach, we only need to generate four twodimensional tables independent of the input combination and slew rate.

Using a two-piece output waveform, Qian et al. propose an effective capacitance calculation approach that approximates the output waveform for single-stage gates [10]. The authors calculate the effective capacitance by equating the currents at the gate output when using the driving-point admittance as the load and when using a single effective capacitor as the load. Average currents for both loads models are equated until the gate output 
voltage reaches the $50 \%$ threshold. Qian's effective capacitance approach requires a large number of iterations (e.g., 5 to 10 iterations). It also involves empirical equations that assume fast input transitions and/or require extensive effort to generate characterization tables. Finally, it does not describe any method to handle complex gates.

Kahng and Muddu [11,13] propose a number of effective capacitance algorithms. In their latest approach [13], they state that by using the voltage of output pin of the gate/cell, they can find a non-iterative and fast method for calculating the effective capacitance that accurately matches the output waveforms in a range from $0.3 V_{d d}$ to $0.6 V_{d d}$. In fact, finding the output transition time (from the complex set of equations that these authors present) can be very costly. Furthermore, the driver resistance in their model is a function of the output load and input transition time and can vary greatly. However, the authors use a single value for the driver resistance corresponding to the case that the driver sees the total capacitances of load. This is a major source of error in their proposed approach.

In this paper, we propose an optimal effective capacitance algorithm for both speed and accuracy; such that this capacitance value approximates both the transition time from 0 to $0.8 \mathrm{Vdd}$ and $50 \%$ propagation delay simultaneously. We also propose a new algorithm to find $50 \%$ propagation delay and " 0 to $0.8 V_{d d}$ " output transition time using effective capacitance for complex gates.

\section{A New Algorithm for Effective Capacitance Calculation of RC Loads}

We now propose a new algorithm that provides the effective capacitance in VDSM technologies, which approximates both the " 0 to $0.8 V_{d d}$ " output transition time and the $50 \%$ gate propagation delay simultaneously.

For any combination of input transition time and output capacitive load, the gate can be replaced with a Thevenin equivalent circuit. This consists of an effective driver resistance, ramp input source and an intrinsic delay [2]. Therefore, the circuit in Fig. 4 can be approximated with the circuit in Fig. 5. Then $R_{d}$ is a linear resistance, which can be written as:

$$
R_{d}=\left\{\begin{array}{lll}
\frac{r_{d n}}{W_{n}} & \text { for } & \text { NMOS } \\
\frac{r_{d p}}{W_{p}} & \text { for } & \text { PMOS }
\end{array}\right.
$$

where $r_{d p}$ and $r_{d n}$ are both functions of input transition time and output capacitive load. $r_{d p}$ and $r_{d n}$ for some combinations of $C_{e f f}$ and $T_{R}$ are shown in table 1 . To find the effective capacitance, what we need is to approximate the $R C-\pi$ load with an effective capacitive load (Fig. 6) such that the voltage at the output pin of the gates in both circuits in Figs. 5 and 6 follow each other during output transition.
Table 1: $r_{d n}$ and $r_{d p}$ for some combinations of $C_{\text {eff }}$ and $T_{R}$

\begin{tabular}{cccc}
\hline$T_{R}$ & $C_{\text {load }}$ & $r_{d n}$ & $r_{d p}$ \\
\hline $200 \mathrm{pS}$ & $200 \mathrm{fF}$ & $23.9 \mathrm{~K}$ & $49.2 \mathrm{~K}$ \\
$200 \mathrm{pS}$ & $500 \mathrm{fF}$ & $14.0 \mathrm{~K}$ & $38.9 \mathrm{~K}$ \\
$400 \mathrm{pS}$ & $200 \mathrm{fF}$ & $34.1 \mathrm{~K}$ & $69.2 \mathrm{~K}$ \\
$300 \mathrm{pS}$ & $500 \mathrm{fF}$ & $15.9 \mathrm{~K}$ & $39.1 \mathrm{~K}$ \\
$100 \mathrm{pS}$ & $300 \mathrm{fF}$ & $12.8 \mathrm{~K}$ & $42.6 \mathrm{~K}$ \\
& & & \\
\hline
\end{tabular}

Figure 5: The equivalent circuit of a gate/cell driving an $R C-\pi$ load.

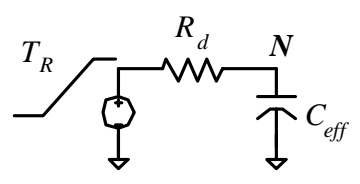

Figure 6: Using $C_{\text {eff }}$ instead of load such that the voltage of $M$ and $N$ in figure 5 and this figure, follow each other during output transition.

The voltages of $M$ and $N$ in Laplacian domain can be written as:

$$
\begin{aligned}
& V_{M}(s)=\frac{s R_{\pi} C_{2}+1}{s^{2} R_{d} R_{\pi} C_{1} C_{2}+s\left[R_{d}\left(C_{1}+C_{2}\right)+R_{\pi} C_{2}\right]+1} V_{\text {in }}(s) \\
& V_{N}(s)=\frac{1}{s R_{d} C_{\text {eff }}+1} V_{\text {in }}(s)
\end{aligned}
$$

where;

$$
V_{\text {in }}(s)=\frac{1-e^{-T_{R} s}}{s^{2}} \frac{V_{d d}}{T_{R}}
$$

Doing inverse Laplace transforms, we can find $V_{M}(t)$ and $V_{N}(t)$ in time domain as follows:

$$
\begin{aligned}
& V_{M}(t)=\left\{\begin{array}{cc}
\frac{V_{d d}}{T_{R}}\left(t-B+A e^{-\alpha t} \operatorname{Cosh}(\omega t+\phi)\right) & 0 \leq t \leq T_{R} \\
\frac{V_{d d}}{T_{R}}\left(T_{R}+A^{\prime} e^{-\alpha t} \operatorname{Cosh}\left(\omega t+\phi^{\prime}\right)\right) & T_{R}<t
\end{array}\right. \\
& V_{N}(t)= \begin{cases}\left.\frac{V_{d d}\left(t-R_{d} C_{e f f}+R_{d} C_{e f f} e^{-\frac{t}{R_{d} C_{e f f}}}\right)}{}\right) & 0 \leq t \leq T_{R} \\
\frac{V_{d d}}{T_{R}}\left(T_{R}+R_{d} C_{e f f}\left(1-e^{\frac{T_{R}}{R_{d} C_{e f f}}}\right) e^{\frac{t}{R_{d} C_{e f f}}}\right. & T_{R}<t\end{cases}
\end{aligned}
$$

where;

$$
\phi=\operatorname{tgh}^{-1}\left(\frac{R_{d}\left(C_{1}+C_{2}\right)^{2}+R_{\pi} C_{2}\left(C_{2}-C_{1}\right)}{\left(C_{1}+C_{2}\right) \sqrt{R_{d}^{2}\left(C_{1}+C_{2}\right)^{2}+2 R_{d} R_{\pi} C_{2}\left(C_{2}-C_{1}\right)+R_{\pi}^{2} C_{2}^{2}}}\right)
$$




$$
\begin{aligned}
& A=\frac{R_{d}\left(C_{1}+C_{2}\right)}{\operatorname{Cosh}(\phi)}, B=R_{d}\left(C_{1}+C_{2}\right), \alpha=\frac{R_{d}\left(C_{1}+C_{2}\right)+R_{\pi} C_{2}}{2 R_{d} R_{\pi} C_{1} C_{2}} \\
& \omega=\frac{\sqrt{R_{d}^{2}\left(C_{1}+C_{2}\right)^{2}+R_{\pi}^{2} C_{2}^{2}+2 R_{d} R_{\pi} C_{2}\left(C_{2}-C_{1}\right)}}{2 R_{d} R_{\pi} C_{1} C_{2}}
\end{aligned}
$$

An accurate approximation for $C_{\text {eff }}$ may be obtained by minimizing the error between voltages of $V_{M}(t)$ and $V_{N}(t)$ from $0.2 V_{d d}$ to $0.8 V_{d d}$. More precisely, we have:

$$
\frac{\partial}{\partial C e f f} \int_{0.2 V d d}^{0.8 V d d}\left(V_{M}-V_{N}\right)^{2} d V=2 \int_{0.2 V d d}^{0.8 V d d}\left(V_{M}-V_{N}\right) \frac{\partial V_{N}}{\partial C e f f} \quad d V=0
$$

which is too complex to solve analytically. From Eq. (11) and (12) it is obvious that $V_{M}(t)$ and $V_{N}(t)$ are both equal at $t=0$. In Eq. (11), $\alpha$ and $\omega$ are the key parameters that determine the behavior of the output voltage. We note that these parameters do not change before and after $t=T_{R}$. Therefore, if we match $V_{M}(t)$ and $V_{N}(t)$ at some critical time instances before $t=T_{R}$, (say $t_{0-}$ $0.5 \mathrm{Vdd}$ ) and calculate an effective capacitance on that basis, then this effective capacitance will be a good approximation of $\alpha$ and $\omega$ even for $\mathrm{t}>T_{R}$ because $V_{M}(t)$ and $V_{N}(t)$ will continue to be very similar even after $t_{0-0.5 \mathrm{Vdd}}$. Our experimental results have shown that the resulting effective capacitance causes $V_{M}(t)$ and $V_{N}(t)$ to closely follow one another at least until $0.8 V_{d d}$. Consequently, we end up with an iterative equation as follows:

$$
\begin{aligned}
C_{e f f}=f\left(C_{e f f}, R_{d}, t\right)=\frac{-A e^{-\alpha t} \operatorname{Cosh}(\omega t+\phi)+B}{R_{d}\left(1-e^{-\frac{t}{R_{d} C_{e f f}}}\right)} \\
=\left(C_{1}+C_{2}\right) \frac{1-e^{-\alpha t} \frac{\operatorname{Cosh}(\omega t+\phi)}{\operatorname{Cosh}(\phi)}}{\left(1-e^{-\frac{t}{R_{d} C_{e f f}}}\right)}
\end{aligned}
$$

To solve the above equation, we need to update the characterization table. Therefore, the new characterization table should have $r_{d n} / r_{d p}, 50 \%$ propagation time, "0-0.5 $V_{d d}$ " output transition time, and " $0-0.8 V_{d d}$ " output transition time for each combination of $T_{R}$ and $C_{\text {eff. }}$.

For fast convergence of the nonlinear iterative equation, we need a good initial value of effective capacitance. Effective capacitance as a function of $R_{\pi}$ for two different values of $T_{i n}$ and $R_{d}$ is shown in Fig. 7.

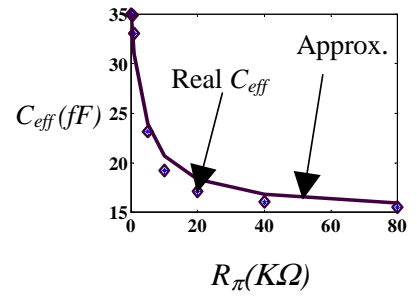

(a)

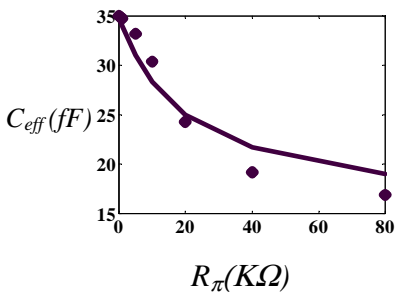

(b)
Figure 7: Effective capacitance for two different cases of $T_{\text {in }}$ and driver sizes $\left(C_{1}=15 f F, C_{2}=20 f F\right)(a)$ driver size $=500 \lambda, T_{R}=100 p S(b)$ driver size $=100 \lambda, T_{R}=200 p S$. $k$ in Eq. (6) is a function of driver strength and resistance of line. If the driver is weak $\left(R_{d}\right.$ is large), the driver would see the entire capacitive load $\left(C_{1}+C_{2}\right)$ and if driver is very strong, then it would see only $C_{l}$, since the output would change very fast and it only sees the near end capacitance. Also, if $R_{\pi}$ goes to infinity, the driver would see only the near end capacitance $\left(C_{l}\right)$ and if $R_{\pi}$ goes to zero, then the driver would see all capacitive load $\left(C_{t o t}=C_{1}+C_{2}\right)$. From the figures and the above explanations, we can say that the initial value, approximately, can be:

$$
C_{e f f}=C_{1}+\frac{R_{d}}{R_{d}+R_{\pi}} C_{2}
$$

which is a very good approximation as an initial guess. $R_{d}$ is the driver resistance when it sees whole capacitive load $\left(C_{\text {eff }}=C_{1}+C_{2}\right)$. Then, using iterative equation, which is shown in Eq. (15), we can find the accurate and fast $C_{\text {eff }}$ for both $50 \%$ propagation delay and $0-0.8 V_{d d}$ output transition time.

Our algorithm for calculating $C_{e f f}$ is as follows. Given the following information for a particular timing path of a cell: the input slew time, $T_{R}$, the $\pi$-load model parameters, $\left(C_{1}, R_{\pi} C_{2}\right)$, and the updated characterized cell output slew model, we perform the following steps:

1. Calculate an initial value of $C_{\text {eff }}$ from Eq. (16).

2. Obtain $t_{0-50 \%}$ from the characterization table based on values of $C_{\text {eff }}$ and $T_{R}$.

3. Obtain $R_{d}$ from the characterization table based on values of $C_{\text {eff }}$ and $T_{R}$.

4. Compute a new value of $C_{\text {eff }}$ from Eq. (15)

5. Find new $t_{0-50 \%}$ based on the new $C_{e f f}$ and given $T_{R}$.

6. Compare the previous value of $t_{0-50 \%}$ with the new $t_{0-50 \%}$ in step 5 .

7. If not within acceptable tolerance, then return to step 3 until $t_{0-50 \%}$ converges.

8. Report $\mathrm{t}_{50 \% \text { propagation delay }}$ and $\mathrm{t}_{0-80 \%}$ from the table.

Using this algorithm, one can obtain an effective capacitance that accurately approximates the $50 \%$ propagation delay as well as output transition time from 0 to $0.8 V_{d d}$.

\section{Extension to Complex Gates}

To use the above algorithm for complex CMOS gates, one must accurately calculate the output resistance of a channel-connected gate. Due to the body effect, it is difficult to compute the exact gate output resistance in channel-connected gates. Let $r_{d n}$ denote the driver resistance of a $1 \mu \mathrm{m}$ wide transistor of length $L$. By using a first order model (usually taught in textbooks on VLSI design), the driver output resistance without consideration of the body effect is calculated by assuming that the "on" resistance of any transistor with a W/L ratio is simply proportional to $L / W$ of that transistor. For instance, the output resistance of a 2-input NAND gate for a falling output transition (with a $W / L$ ratio for each n-type transistor) is simply $2 r_{d n} / W$.

Accounting for the body effect in channel-connected transistors in stacks, Kahng et al. [13] propose an approach with which one can approximate the output resistance more precisely. 
Performing some simulations, they find a body effect coefficient, which is dependent on the number of transistors in the stack and the parameters of the process technology. For instance, the output resistance of a 2-input NAND gate for a falling output transition (with $W / L$ ratios for each n-type transistor) is $2.86 r_{d n} /$ $W$ in $0.25 \mu \mathrm{m}$ process technology. In Kahng's approach, the coefficient is constant for a gate and is independent of the input combination (i.e., which input switches). Consider four input transition cases of a 3 -input NAND gate as shown in Fig. 8. According to both the first order model and Kahng's model, all of these cases result in the same gate output resistance, which is obviously quite inaccurate.

We propose an output resistance calculation equation that uses $m+l$ coefficients for $m$-series-connected transistors in the $N$ or $P$ type stack. More precisely,

$W_{N e f f}=K_{n} \frac{1}{K_{b n(1)} \frac{L_{1}}{W_{1}}+K_{b n(2)} \frac{L_{2}}{W_{2}}+\ldots+K_{b n(m)} \frac{L_{m}}{W_{m}}}$
$W_{P e f f}=K_{p} \frac{1}{K_{b p(1)} \frac{L_{1}}{W_{1}}+K_{b p(2)} \frac{L_{2}}{W_{2}}+\ldots+K_{b p(m)} \frac{L_{m}}{W_{m}}}$

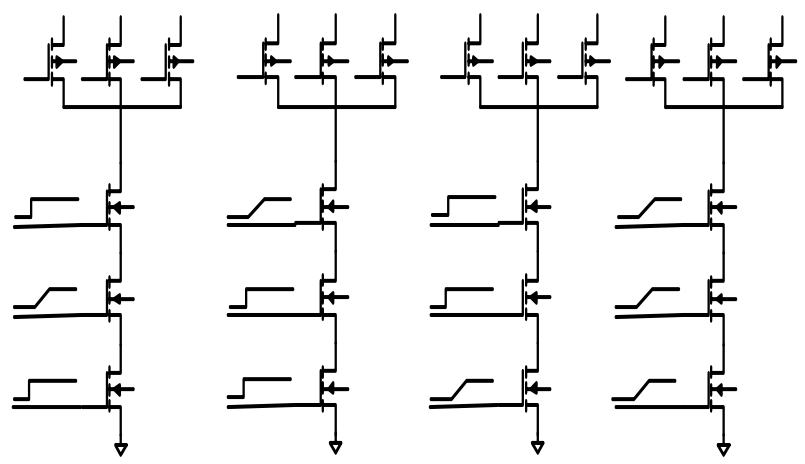

Figure 8: Gate output driver resistance will change according to input pattern.

To find the effective width of channel-connected transistors, we set $K_{b p(i)}$ and $K_{b n(i)}$ in Eq. (17) equal to 1 if the corresponding transistor input does not switch; otherwise, we use tables 2 and 3 to provide the required coefficient values in Eq. (17). $K_{b p}$ 's and $K_{b n}$ 's are obtained by HSPICE simulation for each process technology. For example, for a $0.1 \mu \mathrm{m}$ technology, these values are provided in tables 2 and 3 .

\section{Experimental Results}

We performed simulations for a number of different circuits in $0.1 \mu \mathrm{m}$ CMOS technology and report results. For our experiments we considered two inverters connected in series. We fixed the size of the first inverter at $60 / 30 \mu \mathrm{m}$ and applied an input transition time of $300 p S$ at the its input. We put an RC- $\pi$ load for the second inverter. We provide the results for comparison between HSPICE results and results of our algorithm for the output voltage of the second inverter in table 4 . We also simulate a 3-input NAND gate to validate our algorithm proposed for complex gates. For each input of the 3-input NAND gate, we provided the same circuit as we mentioned for Inverter gate. For Table 5, we only applied the switching input to the top transistor in stack. Table 6 shows the results when we apply a transitioning input waveform with the same slew times to all three inputs of the 3-input NAND gate. All transistors of the same type have the same width as indicated in the first column of the tables. Results show that our approach is both accurate and fast for VDSM technologies within $5 \%$ for both $50 \%$ propagation delay and the " 0 - $0.8 V_{d d}$ " output transition time with at most 3 iterations.

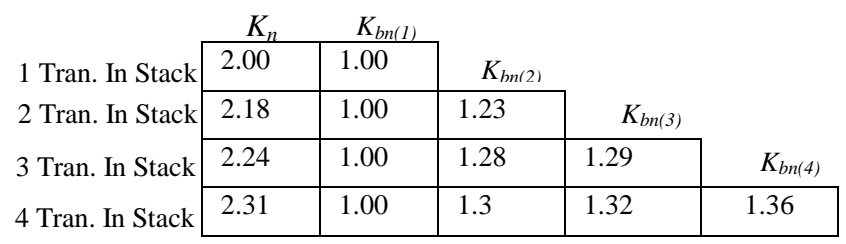

Table 2: Body effect coefficients for $0.1 \mu \mathrm{m}$ technology for NMOS transistors

\begin{tabular}{|c|c|c|c|c|c|}
\hline \multirow{3}{*}{$\begin{array}{l}1 \text { Tran. In Stack } \\
2 \text { Tran. In Stack }\end{array}$} & $K_{p}$ & $K_{b p(1)}$ & \multirow[b]{2}{*}{$K_{b p(2)}$} & \multirow[b]{3}{*}{$K_{b p(3)}$} & \multirow[b]{4}{*}{$K_{b p(4)}$} \\
\hline & 2.00 & 1.00 & & & \\
\hline & 2.26 & 1.00 & 1.35 & & \\
\hline 3 Tran. In Stack & 2.36 & 1.00 & 1.37 & 1.39 & \\
\hline 4 Tran. In Stack & 2.45 & 1.00 & 1.39 & 1.41 & 1.48 \\
\hline
\end{tabular}

Table 3: Body effect coefficients for $0.1 \mu \mathrm{m}$ technology for PMOS transistors

\section{Conclusion}

An increase in interconnect resistance in VDSM technologies has two important results: 1) the interconnect RC-delay resistance portion of a timing path increases and 2) the interconnect resistance reduces the cell delay via shielding the far end capacitances. Gate load delay calculation requires accuracy, and using delay tables is essential for accurate delay calculation for given capacitive load and input transition time. To use the delay tables, what we need is to approximate the load with an effective capacitance that is equivalent to the real load in terms of its $50 \%$ propagation delay and output transition time. In this paper we presented a new efficient algorithm which ends up in an effective capacitance for both $50 \%$ propagation delay and " 0 $0.8 V_{d d}$ " output transition time, simultaneously. In addition, we proposed a new algorithm for complex gates, which calculates the gates delay for different input patterns.

\section{References}

[1] W. C. Elmore, "The Transient Response of Damped Linear Networks with Particular Regard to Wideband Amplifiers," Journal of Applied Physics, 19, Jan. 1948, pp. 55-63.

[2] J. K. Ousterhout, "A Switch-level Timing Verifier for Digital MOS VLSI," IEEE Trans. on Computer Aided Design of VLSI Circuits and Systems, vol. 4 (1985), pp. 336-349.

[3] P. R. O'Brien and T. L. Savarino, "Modeling the DrivingPoint Characteristic of Resistive Interconnect for Accurate Delay Estimation," Proc. of IEEE Int'l Conf. on Computer Aided Design, 1989, pp. 512-515. 
[4] S. P. McCormick, Modeling and Simulation of VLSI Interconnections with Moments, PhD Thesis, MIT, June 1989.

[5] P. R. O'Brien and T. L. Savarino, "Efficient On-Chip Delay Estimation for Leaky Models of Multiple-Source Nets," Proc. of IEEE Custom Integrated Circuits Conf., 1990, pp. 9.6.1-9.6.4.

[6] L. T. Pillage and R. A. Rohrer, "Asymptotic Waveform Evaluation for Timing Analysis," IEEE Trans. on Computer Aided Design of VLSI Circuits and Systems, vol. 9 (1990), pp. 352-366.

[7] C. L. Ratzlaff, N. Gopal, and L. T. Pillage, "RICE: Rapid Interconnect Circuit Evaluator," Proc. of $28^{\text {th }}$ ACM/IEEE Design Automation Conf., June 1991, pp. 555-560.

[8] C. Ratzlaff, S. Pullela, and L. Pillage, "Modeling the RC Interconnect effects in a Hierarchical Timing Analyzer," Proc. of IEEE Custom Integrated Circuits Conference, May 1992, pp. 15.6.1-15.6.4.

[9] M. Sriram and S. M. Kang, "Fast Approximation of the Transient Response of Lossy Transmission Line Trees," Proc. of $30^{\text {th }}$ ACM/IEEE Design Automation Conf., June 1993, pp. 691-696.
[10] J. Qian, S. Pullela, and L. Pillage, "Modeling the "Effective Capacitance" for the RC Interconnect of CMOS gates," IEEE Trans. on Computer Aided Design of VLSI Circuits and Systems, vol. 13 (1994), pp. 1526-1535.

[11] A. B. Kahng and S. Muddu,' “Accurate Analytical Delay Models for VLSI Interconnects," Proc. of IEEE International Symposium on Circuits and Systems, May 1996.

[12]R. Macys, S. McCormick, "A New Algorithm for Computing the "Effective Capacitance" in Deep Submicron Circuits," Proc. of IEEE Custom Integrated Circuits Conference, June 1998, pp. 313-316.

[13] A. B. Kahng and S. Muddu,' 'Improved Effective Capacitance Computations for Use in Logic and Layout Optimization," Proc. of the 12th International Conference on VLSI Design, 1999, Pages: 578 -582.

Table 4: Comparison between our approach with HSPICE for 50\% propagation delay and 0-0.8Vdd for a simple driver in $0.1 \mu \mathrm{m}$ technology

\begin{tabular}{|c|c|c|c|c|c|c|c|c|}
\hline $\begin{array}{l}\text { Inverter Size } \\
(\mathrm{Wp} / \mathrm{Wn}) \mu \mathrm{m}\end{array}$ & $\mathrm{C}_{1}(\mathrm{pF}) / \mathrm{R}_{\pi}(\Omega) / \mathrm{C}_{2}(\mathrm{pF})$ & $\begin{array}{c}\text { HSPICE 50\% } \\
\text { delay (pS) }\end{array}$ & $\begin{array}{c}\text { Estimated } 50 \% \\
\text { delay }(\mathrm{pS})\end{array}$ & Error & $\begin{array}{c}\text { HSPICE } 80 \% \\
\text { delay }(\mathrm{pS})\end{array}$ & $\begin{array}{l}\text { Estimated } 80 \% \\
\text { delay }(\mathrm{pS})\end{array}$ & Error & $\begin{array}{l}\text { Number of } \\
\text { Iterations }\end{array}$ \\
\hline $10 / 5$ & $0.05 / 410 / 0.15$ & 66.1 & 69.0 & $4.5 \%$ & 142.3 & 140.6 & $1.2 \%$ & 3 \\
\hline $40 / 20$ & $0.1 / 290 / 0.25$ & 39.3 & 41.0 & $4.3 \%$ & 95.3 & 97.4 & $2.2 \%$ & 3 \\
\hline $40 / 20$ & $0.5 / 810 / 0.7$ & 74.0 & 76.4 & $3.2 \%$ & 136.2 & 134.5 & $1.3 \%$ & 2 \\
\hline $30 / 15$ & $0.4 / 1000 / 0.8$ & 76.3 & 79.4 & $4.1 \%$ & 142.5 & 138.5 & $2.8 \%$ & 1 \\
\hline $100 / 50$ & $0.9 / 300 / 1.4$ & 62.7 & 65.1 & $3.8 \%$ & 121.0 & 123.1 & $1.7 \%$ & 2 \\
\hline Avg. Error & ------- & ------- & ------- & $4.0 \%$ & ------- & ------- & $1.8 \%$ & ------- \\
\hline
\end{tabular}

Table 5: Comparison between our approach with HSPICE for 50\% propagation delay and 0-0.8Vdd for 3 input NAND gates in $0.1 \mu \mathrm{m}$ technology (when we apply a switching input to the topmost transistor in the stack)

\begin{tabular}{|c|c|c|c|c|c|c|c|c|}
\hline $\begin{array}{l}\text { 3-input NAND } \\
(\mathrm{Wp} / \mathrm{Wn}) \mu \mathrm{m}\end{array}$ & $\mathrm{C}_{1}(\mathrm{pF}) / \mathrm{R}_{\pi}(\Omega) / \mathrm{C}_{2}(\mathrm{pF})$ & $\begin{array}{l}\text { HSPICE } 50 \% \\
\text { delay }(\mathrm{pS})\end{array}$ & $\begin{array}{c}\text { Estimated } 50 \% \\
\text { delay }(\mathrm{pS})\end{array}$ & Error & $\begin{array}{l}\text { HSPICE } 80 \% \\
\text { delay }(\mathrm{pS})\end{array}$ & $\begin{array}{c}\text { Estimated } 80 \% \\
\text { delay (ps) }\end{array}$ & Error & $\begin{array}{l}\text { Number of } \\
\text { Iterations }\end{array}$ \\
\hline $20 / 60$ & $0.4 / 1000 / 0.8$ & $34.7 \mathrm{p}$ & $36.4 p$ & $4.9 \%$ & $42.1 \mathrm{p}$ & $43.2 p$ & $2.6 \%$ & 2 \\
\hline $40 / 120$ & $0.5 / 510 / 1.2$ & $26.4 p$ & $27.1 \mathrm{p}$ & $2.7 \%$ & $78.1 \mathrm{p}$ & $79.5 p$ & $1.8 \%$ & 2 \\
\hline Avg. Error & ------- & ------ & ------- & $3.6 \%$ & ------- & ------- & $2.2 \%$ & ------- \\
\hline
\end{tabular}

Table 6: Comparison of our approach with HSPICE for $50 \%$ propagation delay and $0-0.8$ Vdd for 3 input NAND gate in $0.1 \mu \mathrm{m}$ technology (when we simultaneously apply the same switching input to all inputs)

\begin{tabular}{|c|c|c|c|c|c|c|c|c|}
\hline $\begin{array}{l}\text { 3-input NAND } \\
(\mathrm{Wp} / \mathrm{Wn}) \mu \mathrm{m}\end{array}$ & $\mathrm{C}_{1}(\mathrm{pF}) / \mathrm{R}_{\pi}(\Omega) / \mathrm{C}_{2}(\mathrm{pF})$ & $\begin{array}{c}\text { HSPICE 50\% } \\
\text { delay }(\mathrm{pS})\end{array}$ & $\begin{array}{l}\text { Estimated } 50 \% \\
\text { delay }(\mathrm{pS})\end{array}$ & Error & $\begin{array}{l}\text { HSPICE } 80 \% \\
\text { delay }(\mathrm{pS})\end{array}$ & $\begin{array}{c}\text { Estimated } 80 \% \\
\text { delay }(\mathrm{pS})\end{array}$ & Error & $\begin{array}{l}\text { Number of } \\
\text { Iterations }\end{array}$ \\
\hline $20 / 60$ & $0.4 / 1000 / 0.8$ & $64.7 \mathrm{p}$ & $67 p$ & $3.6 \%$ & $64.4 \mathrm{p}$ & $64.8 \mathrm{p}$ & $0.6 \%$ & 3 \\
\hline $40 / 120$ & $0.5 / 510 / 1.2$ & $54.1 \mathrm{p}$ & $55.5 \mathrm{p}$ & $2.6 \%$ & $83.5 \mathrm{p}$ & $84.5 p$ & $1.2 \%$ & 2 \\
\hline Avg. Error & ------- & ------- & ------- & $3.1 \%$ & ------- & ------- & $0.9 \%$ & ------- \\
\hline
\end{tabular}

